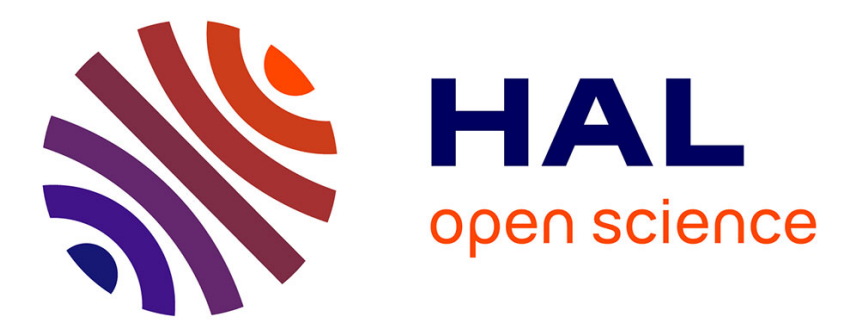

\title{
Navigation in a multi-obstacle environment. from partition of the space to a zonotopic-based MPC
}

Daniel Ioan, Sorin Olaru, Ionela Prodan, Florin Stoican, Silviu-Iulian Niculescu

\section{- To cite this version:}

Daniel Ioan, Sorin Olaru, Ionela Prodan, Florin Stoican, Silviu-Iulian Niculescu. Navigation in a multi-obstacle environment. from partition of the space to a zonotopic-based MPC. ECC 2019 European Control Conference, Jun 2019, Naples, Italy. pp.1772-1777, 10.23919/ECC.2019.8796080 . hal-02328852

\section{HAL Id: hal-02328852}

https://hal-centralesupelec.archives-ouvertes.fr/hal-02328852

Submitted on 10 Apr 2020

HAL is a multi-disciplinary open access archive for the deposit and dissemination of scientific research documents, whether they are published or not. The documents may come from teaching and research institutions in France or abroad, or from public or private research centers.
L'archive ouverte pluridisciplinaire HAL, est destinée au dépôt et à la diffusion de documents scientifiques de niveau recherche, publiés ou non, émanant des établissements d'enseignement et de recherche français ou étrangers, des laboratoires publics ou privés. 


\title{
Navigation in a multi-obstacle environment. From partition of the space to a zonotopic-based MPC.
}

\author{
Daniel Ioan ${ }^{1}$, Sorin Olaru ${ }^{1}$, Ionela Prodan ${ }^{2}$, Florin Stoican ${ }^{3}$, Silviu-Iulian Niculescu ${ }^{1}$
}

\begin{abstract}
This paper pertains to the navigation in a multiobstacle environment and advocates the use of local zonotopic approximations within the obstacle and collision avoidance problem. The design problem is commonly stated in the literature in terms of a constrained optimization problem over a non-convex domain. Firstly, it will be shown that a partition of the navigation space can be obtained using the notion of convex liftings. This partition will offer the foundation for the generation of a path from the current position to the destination point. In order to efficiently describe the navigation on this path, the feasible domain is described using zonotopes. The structural properties of zonotopes with respect to the generic polyhedral sets represents an advantage from the computational point of view. The current paper treats the zonotopic approximations from a control perspective, providing a set of conditions able to safeguard the initial domain topology. Globally, an adaptation of the generic collision avoidance problem is considered, aiming to guarantee the feasibility and highlighting through simulations and proof of concepts illustrations the advantages offered by the use of a zonotopic representation.
\end{abstract}

\section{INTRODUCTION}

The recent proliferation of the real-world applications involving unmanned vehicles has led to a growing interest of the control community on developing reliable and adequate collision-free control strategies within multi-obstacle environment [1], [2].

There exist in the literature two main approaches dealing with the path/trajectory planning in a non-convex feasible space. The optimization-based methods state the problem as a constrained optimization one either over a non-convex domain (e.g. mixed-integer formulation [3], [4]) or with a non-linear cost (e.g. potential field formulation [5], [6]). The second class of approaches, the sampled (or graph) -based methods, replaces the search of feasible paths with the search of the shortest (w.r.t. a predefined criterion) path within a graph whose nodes are randomly selected samples [7], the most popular ones: PRM (probabilistic roadmaps) [8], RRT (rapidly-exploring random tree) [9] and their variants. Moreover, there are some techniques, like tunnel MILP [10], which combines the previously two classes of methods.

Generally, regardless of the approach, a polytopic representation of the obstacles is used. This allows, especially for the first type of methods, an efficient characterization of the non-convex and non-connected feasible domain as

\footnotetext{
${ }^{1}$ Laboratory of Signals and Systems, Univ. Paris-Sud-CentraleSupelecCNRS, Université Paris Saclay. \{daniel.ioan, sorin.olaru, silviu.niculescu\}@l2s. centralesupelec.fr

${ }^{2}$ Univ. Grenoble Alpes, Grenoble INP, LCIS, ionela.prodandlcis.grenoble-inp. fr

${ }^{3}$ Department of Automatic Control and Systems Engineering, UPB, Romania florin.stoicaneacse.pub.ro
}

a union of convex regions through the corresponding hyperplane arrangements in a mixed-integer formalism. The main drawback of this formalism is that the complexity of the control problem is strongly dependent on the number of obstacles. Nonetheless, various technical procedures were developed recently in order to manage the complexity of the formulation: cell merging [11], logarithmic formulations [12] or the use of zonotopic approximations [13].

The later procedure provides rigorous complexity bounds but may lead to infeasibility in the control problem. Therefore, a question appears "How can we approximate the obstacles with zonotopic sets while, simultaneously, safeguarding the feasible paths of the initial problem?". We propose to address this question from two different perspectives. We handle the approximation such that separation among obstacles is preserved (thus avoiding changes in the domain topology and subsequently, infeasibility in the motion planning procedure). In order to identify the obstacles which need to be separated, the problem of finding a feasible trajectory is decomposed into a two-phase algorithm capable to generate feasible trajectories based on a partition of the navigation space. The convex liftings will be instrumental in the construction of a partition while the vertices and faces of the partition will generate a path from the initial state to the destination.

The use of zonotopes is motivated by their intrinsic properties (here, but also in other control areas like: collision detection [14], reachability analysis [15], fault diagnosis [16] or guaranteed state estimation [17]). As well, there are toolboxes like CORA [18] able to manage their representation.

Specifically, the main contributions of this paper are the following:

i) provide a partitioning of the feasible space;

ii) obtain a feasible path;

iii) ensure separation among the zonotopic approximations, i.e., guarding the original space topology;

iv) propose a navigation strategy with obstacle avoidance guarantees using local zonotopic approximations of the obstacles.

The remaining of this paper is divided as follows: Section II presents some basic set-theoretic tools and briefly introduces the formulation of optimization-based control problem, Section III provides a mechanism able to generate feasible geometric paths through the multi-obstacle environment, and Section IV presents a prototype control strategy and treats the zonotopic approximations from the control perspective. Furthermore, Section IV-D proposes a collision 
avoidance strategy and evaluates the results on different scenarios, while Section V draws the conclusions.

Notation: The Minkowski sum of two sets is denoted as $A \oplus B=\{x: x=a+b, a \in A, b \in B\} . \mathcal{C}_{X}(S)$ denotes the complement of the set $S$ over $X \in \mathbb{R}^{d}$, and $\operatorname{int}(S)$ its interior. For $x \in \mathbb{R}^{d}$ we denote $\|x\|_{Q}^{2}=x^{\top} Q x$

\section{PRELIMINARIES}

\section{A. Geometric prerequisites and the basic control problem}

Typically, convex obstacles are modeled using polyhedral sets [4]. Any polytope (i.e. a bounded polyhedron) has a dual representation in terms of intersection of half-spaces:

$$
P=\left\{x \in \mathbb{R}^{d}: s_{i}^{\top} x \leq r_{i}, \forall i\right\} .
$$

or, equivalently, a convex hull of extreme points:

$$
P=\left\{x \in \mathbb{R}^{d}: x=\sum \alpha_{j} v_{j}, \sum \alpha_{j}=1, \alpha_{j} \geq 0, \forall i\right\},
$$

For further use, consider the collection of obstacles (depicted in Fig. 3) as a finite union of polytopes:

$$
\mathbb{P}=\bigcup_{j=1}^{N_{o}} P_{j} .
$$

In order to obtain a collision free trajectory for an agent described by LTI dynamics through a multi-obstacle environment, a MPC (Model Predictive Control) strategy is considered:

$$
\begin{aligned}
\min _{\mathbf{u}}\left(\left\|x_{k+N_{p} \mid k}-\bar{x}_{\mathrm{ref} \mid k}\right\|_{\mathbf{P}}^{2}\right. & +\sum_{l=1}^{N_{p}-1}\left\|x_{k+l \mid k}-\bar{x}_{\mathrm{ref} \mid k}\right\|_{\mathbf{Q}}^{2}+ \\
& \left.+\sum_{l=1}^{N_{p}-1}\left\|\Delta u_{k+l \mid k}\right\|_{\mathbf{R}}^{2}\right)
\end{aligned}
$$

$$
\begin{array}{ll}
\text { s.t. } & x_{k+l \mid k}=A x_{k+l-1 \mid k}+B u_{k+l-1 \mid k}, \\
& x_{k+l \mid k} \in \mathcal{X}, u_{k+l \mid k} \in \mathcal{U}, \\
& x_{k+l \mid k} \notin \mathbb{P} .
\end{array}
$$

The agent dynamics are referenced by (5a) with $x \in \mathbb{R}^{d}$ the state vector, $u \in \mathbb{R}^{d_{u}}$ the input vector and the matrices $A, B$ of appropriate dimension. Furthermore, in (4) - (5) $N_{p}$ is the prediction horizon, the weight matrices $\mathbf{P}$ (terminal cost penalty), $\mathbf{Q}$ (output error penalty) and $\mathbf{R}$ (control move penalty) are positive semi-definite and of appropriate dimensions. The sets $\mathcal{X}$ and $\mathcal{U}$ are compact sets from $\mathbb{R}^{d}$ and $\mathbb{R}^{d_{u}}$, respectively.

While the constraints (5a)-(5b) are inherent in any control problem, the particularity of the anti-collision strategy comes from the non-convex constraints (5c), whose formulation has a substantial impact on the performances and formulation of the control strategy. Hyperplane arrangements are used to characterize these regions. Consider the collection of the support hyperplanes from $\mathbb{R}^{d}$ associated with (3):

$$
\mathbb{H}=\left\{\mathcal{H}_{i}\right\}_{i \in \mathcal{I}}
$$

with $\mathcal{H}_{i}=\left\{x \in \mathbb{R}^{d}: s_{i}^{\top} x=r_{i}\right\}$. Each of these hyperplanes divides the space in two disjoint regions:

$$
\begin{aligned}
& \mathcal{R}_{i}^{+}=\left\{x \in \mathbb{R}^{d}: \quad s_{i}^{\top} x \leq r_{i}\right\}, \\
& \mathcal{R}_{i}^{-}=\left\{x \in \mathbb{R}^{d}:-s_{i}^{\top} x \leq-r_{i}\right\} .
\end{aligned}
$$

Hence, the space can be described as a collection of cells using the hyperplane arrangement notion.

Definition 1 (Hyperplane arrangements - [19]): The collection $\mathbb{H}$ partitions the space into a union of disjoint cells $\mathcal{A}(\sigma)$, characterized by a sign tuple $\sigma \in\{-,+\}^{N}$ :

$$
\mathcal{A}(\sigma)=\bigcap_{i \in \mathcal{I}} \mathcal{R}_{i}^{\sigma(i)} .
$$

The hyperplane arrangement of cells covering the entire space is described by the collection of all feasible sign tuples:

$$
\mathcal{A}(\mathbb{H})=\bigcup_{l=1 \ldots \gamma(N)}\left(\sigma_{l}\right),
$$

where $\sigma_{l} \in\{-,+\}^{N}$ is the sign tuple resulting from a nonempty intersection of half-spaces and $\gamma(N)$ is the number of feasible cells.

Labeling the feasible cells (8) into interdicted $\Sigma_{\mathbb{P}}=\{\sigma$ : $A(\sigma) \cap \mathbb{P} \neq \emptyset\}$ or allowed $\Sigma_{X \backslash \mathbb{P}}=\{\sigma: A(\sigma) \cap \mathbb{P}=\emptyset\}$ we rewrite the MPC problem (4), replacing the constraints $(5 \mathrm{c})$ with:

$$
x_{k+l \mid k} \in \mathcal{A}\left(\sigma_{a}\right), \quad \forall \sigma_{a} \in \Sigma_{X \backslash \mathbb{P}} .
$$

The constraints (10) have the advantage of a inclusion type in comparison with $(5 \mathrm{c})$ but brings the complexity of the enumeration of the components.

\section{B. Motivation and problem statement}

Generally speaking, the performance of the MPC problem (4) covers several aspects:

(a) dynamic performance,

(b) convergence and recursive feasibility,

(c) complexity of the representation and anti-collision guarantees.

These aspects can be dealt with by properly adjusting the tuning parameters appearing in the MPC formulation:

i) $N_{p}$, the prediction horizon,

ii) the value(s) of $\bar{x}_{\text {ref } \mid k}$,

iii) the weight matrices $\mathbf{P}, \mathbf{Q}$ and $\mathbf{R}$.

While the dynamic performance is affected, mainly, by the choice of parameters iii), the aspect (b) strongly depends on the way we choose the parameters i) and ii). Ultimately, complexity and anti-collision feature (c) is correlated with the formal description of the non-convex and non-connected constraints $(5 \mathrm{c})$.

In what follows we focus on providing a control strategy, tackling the control problem (4) from the perspective of objectives (b) and (c). Therefore, we propose a collision avoidance strategy which is built on:

i) construction of a partition of $\mathcal{X} \subseteq \mathbb{R}^{d}$ such that we have a one-to-one relationship with obstacles (Section III-A), 
ii) generation of reference trajectories with feasibility guarantees (Section III-B),

iii) a real-time MPC, enhanced by a zonotopic representation of the multi-obstacle environment (Section IV).

\section{FEASIBLE PATH GENERATION}

\section{A. Space partitioning}

In what follows, we build a workspace partition which relies on the obstacles. We introduce a couple of definitions.

Definition 2: A family of sets $\left\{X_{i}\right\}_{i \in \mathcal{I}}$ verifying:

i) $\mathcal{X}=\bigcup_{i=1}^{N_{o}} X_{i}$,

ii) $\operatorname{int}\left(X_{i}\right) \bigcap \operatorname{int}\left(X_{j}\right)=\emptyset, \forall i \neq j \in \mathcal{I}$,

is called partition of $\mathcal{X} \subseteq \mathbb{R}^{d}$.

Definition 3: If the sets $\mathcal{X}$ and $X_{i}, \forall i$ in Def. 2 are polyhedral, then $\mathcal{X}=\bigcup_{i=1}^{N_{o}} X_{i}$ is called a polyhedral partition.

With these definitions our objective is formulated in terms of constructing $\mathcal{X}=\bigcup_{i=1}^{N_{o}} X_{i}$ with $P_{i} \subset X_{i}$, through a convex lifting method [20].

Definition 4 (Convex lifting [20]): For a polyhedral partition $\left\{X_{i}\right\}_{i \in \mathcal{I}}$ of a polyhedron $\mathcal{X}$, a piecewise affine lifting described by the function:

$$
z(x)=a_{i}^{\top} x+b_{i}, x \in X_{i},
$$

is called a convex (piecewise affine) lifting, if $z(x)$ is continuous and convex over partition $\left\{X_{i}\right\}_{i \in \mathcal{I}}$ of $\mathcal{X}$.

Remark 1: The lifting $z(x)$ satisfies the continuity condition if $a_{i}^{\top} x+b_{i}=a_{j}^{\top} x+b_{j}, \forall x \in X_{i} \cap X_{j}$.

Remark 2: The function $z(x)$ being convex over $\left\{X_{i}\right\}_{i \in \mathcal{I}}$ means that $z(x)>a_{j}^{\top} x+b_{j}, x \in X_{i} \backslash X_{j}, \forall i \neq j \in \mathcal{I}_{N}$

For further use, we denote by $\left\{X_{i}\right\}_{i \in \mathcal{I}}$ the partition obtained through convex lifting, and, for the ease of the presentation, the following assumption is in order.

Assumption 1: The compact set $\mathcal{X}$ is a polyhedron.

The actual construction of the convex lifting is done by solving the convex optimization problem which follows:

$$
\begin{array}{ll}
\min _{a_{i}, b_{i}} & \sum_{i=1}^{N_{o}} J\left(a_{i}, b_{i}\right)=\sum_{i=1}^{N_{o}}\left[\begin{array}{ll}
a_{i}^{\top} & b_{i}
\end{array}\right]\left[\begin{array}{l}
a_{i} \\
b_{i}
\end{array}\right] \\
\text { s.t. } & a_{i}^{\top} v+b_{i} \leq M, \forall v \in \mathcal{V}\left(P_{i}\right), \forall i, \\
& a_{i}^{\top} v+b_{i} \geq a_{j}^{\top} v+b_{j}+\epsilon, \forall v \in \mathcal{V}\left(P_{i}\right), \forall i \neq j, \\
& a_{j}^{\top} \nu+b_{j} \geq a_{i}^{\top} \nu+b_{i}+\epsilon, \forall \nu \in \mathcal{V}\left(P_{j}\right) \forall i \neq j .
\end{array}
$$

where $M$ is a sufficiently large ('big-M") constant, $\epsilon \geq 0$ a given constant, and $\mathcal{V}\left(P_{i}\right)$ stands for the set of the vertices of $P_{i}, \forall i=1: N_{o}$ in (3).

Remark 3: Compared to the standard construction from [20], the problem (12) uses the vertices of the obstacle $P_{i}$ instead of vertices of the partition cell $X_{i}$. Moreover, while the constraint (12b) represents an upper bound for the lifting, the other two constraints are given by the characteristics of the convex lifting, according to Def. 4.

Next, we proceed to obtain the partition $\left\{X_{i}\right\}_{i=1: N_{o}}$ from its convex lifting. Therefore, gathering the solutions of (12), we define the following " $\mathrm{d}+1$ "'-dimensional polyhedron:

$$
\mathcal{P}=\left\{\left[\begin{array}{l}
x \\
z
\end{array}\right] \in \mathbb{R}^{d+1}:\left[a_{i}^{\top}-1\right]\left[\begin{array}{l}
x \\
z
\end{array}\right] \leq-b_{i}, \quad i=1: N_{o}\right\} .
$$

Projecting the facets of $\mathcal{P}$ on $\mathcal{X}$, we obtain the polyhedral partition $\left\{X_{i}\right\}_{i=1: N_{o}}$ :

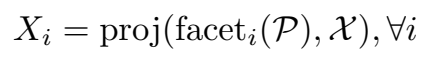

where facet $_{i}(\mathcal{P})$ stands for the $i$-th facet of the polyhedron $\mathcal{P}$, i.e. the inequalities describing $\mathcal{P}$ remains the same except the $i$-th one which is converted in an equality. The properties of the partition obtained in this manner are summarized by the following proposition.

Proposition 1: If problem (12) is feasible, then the partition $\left\{X_{i}\right\}_{i=1: N_{o}}$ has the following properties:

i) $P_{i} \subset X_{i}, \forall i$

ii) $X_{i} \cap P_{j}=\emptyset, \forall j \neq i$.

Proof:

i) The feasibility of (12) implies that (12c) is checked. Thus, considering (2), it means that for all $x \in P_{i}$ we have: $\left[\begin{array}{c}x \\ z(x)\end{array}\right]=\left[\begin{array}{c}x \\ a_{i}^{\top} x+b_{i}\end{array}\right] \in \mathcal{P}$. In other words, the lifting corresponding to $P_{i}$ is included in $\operatorname{facet}_{i}(\mathcal{P})$. By projecting over $X$, it directly leads to the conclusion.

ii) By reduction to the absurd, let us suppose that there is a value $y \in X_{i} \cap P_{j}$. According to i) we have $P_{j} \subset X_{j}$. That leads to $y \in X_{j}$ and, from feasibility of (12c):

$$
a_{j}^{\top} y+b_{j} \geq a_{i}^{\top} y+b_{i}+\epsilon .
$$

Moreover, $y \in X_{j}$ means that $y \in X_{i} \cap X_{j}$ and $\left(X_{i}, X_{j}\right)$-neighboring cells. By consequence, as stated in Remark 1, we have:

$$
a_{i}^{\top} y+b_{i}=a_{j}^{\top} y+b_{j} .
$$

Combining (15) and (16), gives $\epsilon \leq 0$, which contradicts the initial condition $\epsilon>0$.

Remark 4: The feasibility of (12) implies the existence of a convex liftable partition of the workspace $\mathcal{X}$ and provides a versatile tool for the construction of a partition based on the obstacles. It should be mentioned that the infeasibility of (12) does not exclude the existence of either a convex non-liftable or non-convex liftable partition [20].

\section{Illustrative examples}

Fig. 1 depicts the partition cells for two different multiobstacle environments. We note that there exist a one-to-one relationship between obstacles and partition cells. As a side remark, the construction based on lifting is extremely fast and, thus, can be evaluated for moving obstacles (however, this case is out of the scope of the present paper).

\section{B. Geometric path generation}

The constructive result from the previous subsection holds for $\mathbb{R}^{d}$. For the clarity of the presentation, in this subsection we restrict the scope to the $d=2$ case and aim to guarantee a path for any pair of initial and final points in the workspace.

For the purpose of obtaining collision-free geometric path through a multi-obstacle environment, we define a weighted graph $\Gamma=(\mathcal{N}, \mathcal{E}, f), f: \mathcal{E} \rightarrow \mathbb{R}$, based on the partition 


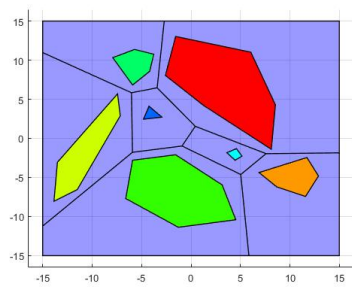

(a) $N_{o}=7$

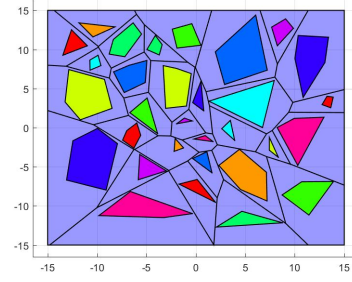

(b) $N_{o}=31$
Fig. 1: Space-partitioning as in (14).

$\left\{V_{i}\right\}_{i=1: N_{o}}$ of the workspace $\mathcal{X}$. Hence, the nodes of $\Gamma$ are the vertices of the polyhedral cells:

$$
\mathcal{N}=\bigcup_{i=1}^{N_{o}} \mathcal{V}\left(X_{i}\right)
$$

the edges $\mathcal{E}$ are the hyperplanes connecting the selected vertices (i.e. the facets of the partition cells), and the function $f$ is given by the Euclidean distance between the incident nodes of the edge. For illustration, we have depicted in Fig. 2, the graph corresponding to the polyhedral partition in Fig. $1 \mathrm{~b}$.

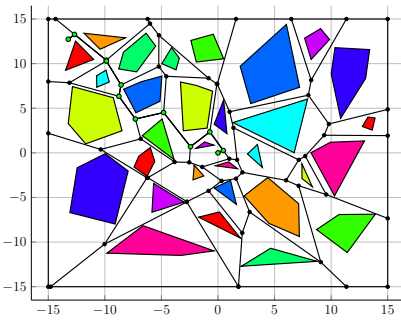

(a) $\tilde{\Gamma}(\mathcal{N}, \mathcal{E}, f)$

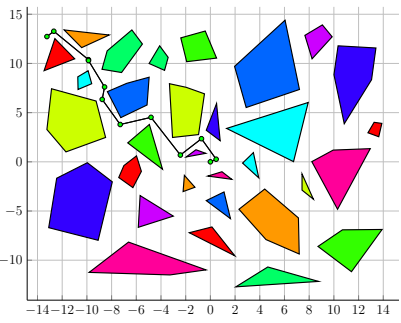

(b) $\operatorname{Path}\left(x_{i}, x_{f}\right)$
Fig. 2: Graph $\Gamma$ and the shortest path $\operatorname{Path}\left(x_{i}, x_{f}\right)$.

\section{Proposition 2: $\Gamma$ is a connected planar graph.}

Proof: Since $\left\{X_{i}\right\}_{i=1: N_{o}}$ is a partition of $\mathcal{X}$ having the properties described in Def. 2 , it results that there exists no intersection among the facets of partitions cells $V_{i}$, except the vertices, and, by consequence, among edges of the graph (i.e. the graph is planar). Moreover, there exists a sequence of facets which links any two vertices of the partition $\left\{X_{i}\right\}$. Hence, there exists a path through the graph between any two nodes in $\mathcal{N}$ (i.e. the graph is connected).

Finding the shortest path through the graph between the start and final points $x_{i}, x_{f} \in \mathcal{X} \backslash \mathbb{P}$ means adding them to the graph and finding the closest edge such that the connection to it does not intersect any obstacles:

$$
\begin{aligned}
\left(j^{\perp}, x^{\perp}\right)\left(x_{i}\right)=\arg \min _{j \in \mathcal{I}_{f_{i}} x \in \operatorname{macet}_{j}\left(X_{i}\right)}\left\|x-x_{i}\right\| \\
\text { s.t. } \quad \alpha x+(1-\alpha) x_{i} \notin P_{i}, \quad x_{i} \in X_{i},
\end{aligned}
$$

where $\mathcal{I}_{f_{i}}$ is the number of facets of $X_{i}$. These projections of the points over the closest edges are also new nodes of the graph. Thus, the graph $\Gamma$ properties are preserved by the new graph $\tilde{\Gamma}\left(x_{i}, x_{f}\right)$. Afterward, on account of
$\tilde{\Gamma}\left(x_{i}, x_{f}\right)$ properties, a graph search algorithm (e.g. Dijkstra's Algorithm [8]) is employed and the shortest path between $x_{i}$ and $x_{f}$ is obtained. For example, in Fig. 2, the green nodes are the components of the shortest path.

Remark 5: The existence of a shortest path through the graph $\tilde{\Gamma}\left(x_{i}, x_{f}\right)$ between any pair $\left(x_{i}, x_{f}\right) \in(\mathcal{X} \backslash \mathbb{P}) \times(\mathcal{X} \backslash \mathbb{P})$ is guaranteed for the reason that the the graph $\tilde{\Gamma}\left(x_{i}, x_{f}\right)$ is connected for any pair $\left(x_{i}, x_{f}\right)$. For further use, we denote the shortest path between $x_{i}$ and $x_{f}$ as $\operatorname{Path}\left(x_{i}, x_{f}\right)=$ $\left(\bar{x}_{0}=x_{i}, \bar{x}_{1}=x^{\perp}\left(x_{i}\right), \ldots, \bar{x}_{n}=x^{\perp}\left(x_{f}\right), \bar{x}_{n+1}=x_{f}\right)$, the ordered set of points forming the shortest path.

\section{FROM POLYHEDRAL TO ZONOTOPIC MPC FOR COLLISION AVOIDANCE}

Let us consider the feasible path $\operatorname{Path}\left(x_{i}, x_{f}\right)$ through the environment given by the method detailed in Section III. Our aim is to incorporate it in a optimization-based collision avoidance strategy, e.g, the MPC problem (4).

\section{A. Prototype polytopic MPC for collision avoidance}

Firstly, we propose a motion planning strategy which exploits, besides the existence of a feasible path, the polytopic representation of the obstacles and offers feasibility guarantees. To this end, we rewrite (4) in a simpler form:

$$
\begin{aligned}
& u_{M P C_{3}}^{*}= \arg \min \left(\left\|x_{k+1 \mid k}-\bar{x}_{1 \mid k}\right\|_{Q_{1}}^{2}\right. \\
&\left.+\left\|x_{k+2 \mid k}-\bar{x}_{2 \mid k}\right\|_{Q_{2}}^{2}+\left\|x_{k+3 \mid k}-\bar{x}_{3 \mid k}\right\|_{Q_{3}}^{2}\right) \\
& \text { s.t. } \quad x_{k+l \mid k}=A x_{k+l-1 \mid k}+B u_{k+l-1 \mid k}, \\
& x_{k+l \mid k} \in \mathcal{X}, u_{k+l \mid k} \in \mathcal{U}, \\
& x_{k+l \mid k} \notin \tilde{\mathbb{P}}(k)
\end{aligned}
$$

where $\tilde{\mathbb{P}}(k)=\left\{P_{i} \in \mathbb{P}: i\right.$ s.t. $\left.\operatorname{Path}\left(x_{k}, x_{f}\right) \cap X_{i} \neq \emptyset\right\}$ is a significantly reduced set of obstacles. Form (19) provides feasibility guarantees but does not certify the convergence. This feature can be enforced if we consider two more MPC problems with the same constraints as in (19):

$$
\begin{aligned}
& u_{M P C_{2}}^{*}=\arg \min \left(\left\|x_{k+1 \mid k}-\bar{x}_{1 \mid k}\right\|_{Q_{1}}^{2}\right. \\
& \left.+\left\|x_{k+2 \mid k}-\bar{x}_{2 \mid k}\right\|_{Q_{2}}^{2}\right), \\
& u_{M P C_{1}}^{*}=\arg \min \left(\left\|x_{k+1 \mid k}-\bar{x}_{1 \mid k}\right\|_{Q_{1}}^{2}\right),
\end{aligned}
$$

and select the input to be applied via optimization (22):

$$
\begin{aligned}
& u_{M P C}^{*}=\max _{i=1: 3} i \\
& \text { s.t. }\left(x^{\perp}\left(x_{k+1}^{*}\right), \operatorname{proj}\left(x^{\perp}\left(x_{k+1}^{*}\right), \text { facet }_{i \perp}\left(X_{k}\right)\right)\right) \cap P_{i} \neq \emptyset
\end{aligned}
$$

where $x_{k+1}^{*}=A x_{k}+B u_{M P C_{i}}^{*}$.

Theorem 1: If $\operatorname{Path}\left(x_{k}, x_{f}\right)$ exists and the control law $u_{M P C}$ in (22) is recursive feasible then it guarantees the convergence $x_{k} \rightarrow x_{f}$.

Sketch of Proof: All the points $\bar{x}_{i \mid k}$ are either on the boundary of $X_{k}$ or outside of it. The cost function is designed to minimize the tracking error with respect to three points and, thus, in the absence of constraints (19b)(19c), the trajectory will leave $X_{k}$, crossing the boundary in finite time as long as $x_{f} \notin X_{k}$. The recursive feasibility 
assumption ensures the compatibility of (19b). However, (19c) can threaten the convergence if the state converges to a point on the boundary of $P_{k}$. The existence of a trajectory converging to the borders of $X_{k}$ is guaranteed by the existence of $\operatorname{Path}\left(x_{k}, x_{f}\right)$. Nevertheless, this trajectory might not be generated by $u_{M P_{3}}^{*}$ which weights the tracking at multiple stages and, thus, violating (22b). In order to enforce the satisfaction of (22b), the receding optimization will be enhanced with $u_{M P C_{2}}^{*}$ and $u_{M P C_{1}}^{*}$. The last one represents an explicit choice of a control converging to the boundary of $X_{k}$. Thus, the feasibility of (22b) preserves the subsequence of edges in $\operatorname{Path}\left(x_{k}, x_{f}\right)$ and the path length (in terms of Euclidean distance) will play the role of a candidate Lyapunov function.

Remark 6: Constraint $x \notin \tilde{\mathbb{P}}(k)$, is reformulated as an inclusion, as in (10). Thus, the complexity of enumeration is substantially reduced, i.e. the number of cells generated by the reduced set of hyperplanes is significantly smaller compared to the one generated in the general case (6).

Hereinafter, the number of anti-collision constraints is reduced in term of number of obstacles and the only computational advantage can be obtained by decreasing the complexity of the obstacles' representation. Thus, in the next subsections we will use a particular class of polytopes, commonly known as zonotopes, which are endowed with a third representation due to their generic symmetry property.

\section{B. Zonotopic approximations and complexity bounds.}

Definition 5 (Zonotopes-[21]): A zonotope is a centrally symmetric polytope, which can be described as a Minkowski sum of line segments. In its generator representation a zonotope $\mathcal{Z}(G, c)$ is described by center $c \in \mathbb{R}^{d}$ and generators $G=\left[\begin{array}{lll}g_{1} & \cdots & g_{m}\end{array}\right] \in \mathbb{R}^{d \times m}$ :

$$
\mathcal{Z}(G, c)=\left\{c+\sum_{i=1}^{m} \xi_{i} g_{i}:\|\xi\|_{\infty} \leq 1\right\}
$$

Zonotopes own several properties of practical interest [22]:

i) are closed under Minkowski sum:

$$
\mathcal{Z}\left(G_{1}, c_{1}\right) \oplus \mathcal{Z}\left(G_{2}, c_{2}\right)=\mathcal{Z}\left(\left[\begin{array}{ll}
G_{1} & G_{2}
\end{array}\right], c_{1}+c_{2}\right)
$$

ii) are symmetric, up to their center:

$$
-\mathcal{Z}\left(G_{1}, c_{1}\right)=\mathcal{Z}\left(G_{1},-c_{1}\right) \text {. }
$$

The half-space representation can be obtained from the generator form of a zonotope (23), as follows [15]: to each sequence of $d-1$ generators $1 \leq j_{1}<j_{2} \ldots j_{d-1} \leq m$ corresponds the pair $\left(h_{i}, k_{i}\right) \in \mathbb{R}^{d} \times \mathbb{R}$, where:

$$
h_{i} \perp g_{j_{l}}, \forall j_{l} \in\left\{j_{1} \ldots j_{d-1}\right\}, k_{i}=\sum_{j_{l} \notin\left\{j_{1} \ldots j_{d-1}\right\}}\left|h_{i}^{\top} g_{j_{l}}\right| \text {. }
$$

Adding the center from generator representation, a zonotope is formulated as:

$$
\mathcal{Z}(G, c)=\bigcap_{1 \leq j_{1}<\ldots j_{d-1} \leq m}\left\{x \in \mathbb{R}^{d}:\left|h_{i}(x-c)\right| \leq k_{i}\right\} .
$$

Moreover, using the support function [23] (and its interesting application regarding the inclusion condition) in combination with the definition of a zonotope, the inclusion $\mathcal{Z}(c, G) \subseteq P$, with $P$ defined as in (1), is checked iff:

$$
s_{i}^{\top} c+\sum_{j}\left|s_{i}^{\top} g_{j}\right| \leq r_{i} \quad \forall i, j .
$$

Moreover, the inclusion of a polytopic set $P$, defined as in (2), into a zonotope $\mathcal{Z}(G, c)$ holds iff

$$
\left|h_{i}^{\top}\left(v_{j}-c\right)\right| \leq k_{i} \quad \forall i, j .
$$

Considering Def. 5 and (23) we refer to a family of zonotopes parametrized after their centers $c_{\ell} \in \mathbb{R}^{d}$ and scaling factors $\Delta_{\ell} \in \mathbb{R}^{m \times m}$ applied to a common generator "seed" (an a priori given matrix $G \in \mathbb{R}^{d \times m}$ ):

$$
\mathbb{Z}=\left\{\mathcal{Z}\left(G \Delta_{j}, c_{j}\right), \quad j=1 \ldots N_{o}\right\}
$$

$\Delta_{\ell}$ is a diagonal matrix whose diagonal elements are equal $/$ distinct $^{1}$. The $\mathrm{k}$-th diagonal element is noted as $\delta_{j_{k}}$. This approach threads the line between complexity and feasibility.

Replacing $g_{k}$ with $g_{k} \cdot \delta_{j_{k}}$ in (26), the half-space representation of the $\mathrm{j}$-th zonotope from (30) is given by:

$$
\begin{aligned}
h_{i} \text { s.t. } h_{i} \perp g_{k}, \forall k \in\left\{k_{1} \ldots k_{d-1}\right\}, \\
k_{i}\left(\Delta_{j}\right)=\sum_{k \notin\left\{k_{1} \ldots k_{d-1}\right\}}\left|h_{i}^{\top} g_{k}\right| \delta_{j_{k}},
\end{aligned}
$$

where $i$ enumerates the

$$
p(d, m)=\left(\begin{array}{c}
m \\
d-1
\end{array}\right)
$$

combinations of $d-1$ distinct generators selected from the list of $m$ available ones (i.e., $1 \leq k_{1}<\cdots<k_{d-1} \leq m$ ).

For further use we gather the support hyperplanes resulted from (31) into the collection

$$
\tilde{\mathbb{H}}=\left\{\mathcal{H}\left(h_{i}, \pm k_{i}\left(\Delta_{j}\right)\right)\right\}, \forall j=1 \ldots N_{o}, i=1 \ldots p(d, m)
$$

Parametrization (31), with set $P$ defined as before (1)-(2), allows to reformulate the inclusion conditions (28), (29) in a linear form with respect to parameters $c_{j}, \Delta_{j}$ :

$$
\begin{aligned}
& \mathcal{Z}\left(G \Delta_{j}, c_{j}\right) \subseteq P: s_{i}^{\top} c_{j}+\sum_{k=1}^{m}\left|s_{i}^{\top} g_{k}\right| \cdot \delta_{j_{k}} \leq r_{i}, \forall i, \\
& P \subseteq \mathcal{Z}\left(G \Delta_{j}, c_{j}\right):\left|h_{i}^{\top}\left(v_{k}-c_{j}\right)\right| \leq k_{i}\left(\Delta_{j}\right), \forall k .
\end{aligned}
$$

The overall goal is to provide adequate over-approximations (30) for the multi-obstacle environment (3). That is, seek a (inherently symmetric) zonotope $\mathcal{Z}\left(G \Delta_{j}, c_{j}\right)$ enclosing the known (usually non-symmetric) polytope $P_{j}$ such that a specific measure parametrized after $c_{j}, \Delta_{j}$ is minimized:

$$
\begin{aligned}
\left(\Delta_{j}, c_{j}\right)^{*}= & \arg \min _{\Delta_{j}, c_{j}} \mathcal{C}\left(\Delta_{j}, c_{j}\right) \\
& \text { s.t. } P_{j} \subseteq \mathcal{Z}\left(G \Delta_{j}, c_{j}\right) .
\end{aligned}
$$

Although several measures can be considered [] with different performances, in this paper we opted to use the measure

\footnotetext{
${ }^{1}$ If not explicitly stated otherwise, we consider the later case since the former is a simplification of the later.
} 
which provides the most accurate (tightest) approximation, specifically the one given by the volume (see the Appendix):

$$
\mathcal{C}\left(\Delta_{j}, c_{j}\right)=\sum_{1 \leq k_{1}<\cdots<k_{d} \leq m}\left|\operatorname{det}\left(G^{k_{1} \ldots k_{d}}\right)\right| \cdot \prod_{k \in\left\{k_{1}, \ldots, k_{d}\right\}} \delta_{j_{k}} .
$$

Considering the problem (35) and the collection of obstacles (3), we define the operator:

$$
\mathbb{Z}^{*}(\mathbb{P})=\left\{\mathcal{Z}\left(G \Delta_{j}^{*}, c_{j}^{*}\right), \quad j=1 \ldots N_{o}\right\} .
$$

Collection (33), based on (30) induces the hyperplane arrangement $^{2} \mathcal{A}(\tilde{\mathbb{H}})$. Preliminary work provides a cell count with explicit dependence on $m$, the number of generators and $N_{o}$, the number of obstacles:

$$
\begin{aligned}
& r(\mathcal{A})=\sum_{k=0}^{d}\left(\begin{array}{c}
p(d, m) \\
k
\end{array}\right) \cdot\left(2 N_{o}\right)^{k}, \\
& b(\mathcal{A})=\left|\sum_{k=0}^{d}(-1)^{k}\left(\begin{array}{c}
p(d, m) \\
k
\end{array}\right) \cdot\left(2 N_{o}\right)^{k}\right| .
\end{aligned}
$$

where $r(\mathcal{A})$ is the total number of regions, and $b(\mathcal{A})$ the number of bounded regions. Furthermore, these explicit expressions allow to derive a bound for the number of generators in the zonotopic representation.

Corollary 1: Assuming $n_{o}^{*}$ support hyperplanes in (3), for any $m \in \mathbb{N}^{+}$which verifies:

$$
\sum_{k=0}^{d}\left(\begin{array}{c}
p(d, m) \\
k
\end{array}\right) \cdot\left(2 N_{o}\right)^{k} \leq \sum_{k=0}^{d}\left(\begin{array}{c}
n_{o}^{*} \\
k
\end{array}\right),
$$

the arrangement $\mathcal{A}(\tilde{\mathbb{H}})$ has fewer cells than $\mathcal{A}(\mathbb{H})$.

While Corollary 1 holds for $\mathbb{R}^{d}$, for $d=2$ and $d=3$ there exists a particularization of the results which leads to an analytic formula for the largest number of the generators $m$ such that $\mathcal{A}(\tilde{\mathbb{H}})$ has fewer cells than $\mathcal{A}(\mathbb{H})$. For example, in Fig. 3a we have $N_{o}=7$ hyperplanes and $n_{o}^{*}=34$ supporting hyperplanes. Computing the bounds for the number of cells (38) and using Corollary 1, we obtain the largest value of $m$ for which the approximation is less complex : $m=$ $\lfloor 2.92 \ldots\rfloor=2$. This leads to values $r(\mathcal{A})=225$, significantly less than 407 if the $2 m \cdot N_{o}=28$ hyperplanes would have been in general position and less than 419, the number of cells when considering the initial representation ${ }^{3}$.

\section{Zonotopic approximations with corridors}

However, all the improvements in the complexity, presented in the above subsection, are related only to the geometry and they were not analyzed from the dynamics perspective. For the sake of illustration, we proceed to find the zonotopic approximations $\mathbb{Z}$ for the collection (3) solving the problem (35) for the criterion (36) with an empirically chosen value of the common seed: $G=\left[\begin{array}{ll}1 & 0 \\ 0 & 1\end{array}\right]$. In Fig. 3 we remark that the zonotopic approximations corresponding to $P_{3}$ and $P_{4}$ intersect which may lead to infeasibility in the

\footnotetext{
${ }^{2}$ For compactness, whenever clear from the context we use notation $\mathcal{A}$.

${ }^{3}$ The differences are more significant for $d=3$ but for the clarity of the presentation we tackled here exclusively the $d=2$ case.
}

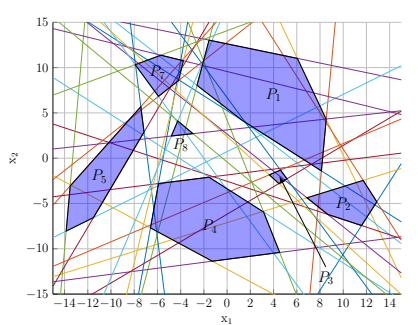

(a) initial

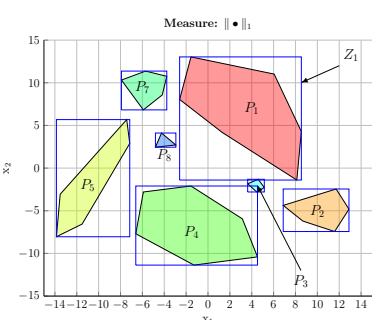

(b) $G=G_{3}$
Fig. 3: Zonotopic approximation for the multi-obstacle environment

control strategy. Hereinafter, we treat the problem of closing corridors or passageways.

Definition 6: A corridor between $i$-th and $j$-th obstacles is called obstructed iff:

$$
P_{i} \cap P_{j}=\emptyset \quad \text { and } \quad Z_{i} \cap Z_{j} \neq \emptyset,
$$

where $Z_{i}, Z_{j}$ represent the zonotopic approximations of $P_{i}$ and $P_{j}\left(P_{i} \subseteq Z_{i}\right.$ and $\left.P_{j} \subseteq Z_{j}\right)$.

There are two possible ways to elude the overlapping and, thus, to maintain the corridors open. Both share a common feature: a separating hyperplane constraint in the optimization problem (35). In the sequel, the hyperplane separating the polytopes $P_{i}$ and $P_{j}$ is denoted as $\mathcal{H}_{i j}=$ $\left\{x \in \mathbb{R}^{d}: h_{i j}^{\top} x=k_{i j}\right\}$, with its corresponding half-spaces $\mathcal{R}_{i j}^{+}$and $\mathcal{R}_{i j}^{-}$. It is obtained from the edges of the partition $\left\{X_{i}\right\}_{i=1: N_{o}}$ in (14), i.e., $\mathcal{H}_{i j}$ represents the common facet between any two neighbor cells of the partitioning $\left(X_{i}, X_{j}\right)$.

The first approach imposes an additional constraint: the approximation to stay above or below a separation hyperplane. The decision between above or below is made based on the position of the center of the polytope relative to this given separation hyperplane. In other words, we impose:

$$
\mathcal{Z}\left(G_{j} \Delta_{j}, c_{j}\right) \subset \mathcal{R}_{i j}^{+} \text {or } \mathcal{Z}\left(G_{j} \Delta_{j}, c_{j}\right) \subset \mathcal{R}_{i j}^{-}, \quad \forall i .
$$

The reformulation of (41) as an $l p$ is straightforward: all vertices of the zonotopic approximation have to stay on the same side of the separation hyperplane as its center.

Remark 7: The vertices of the zonotopes can be readily obtained (28) and the complexity of the dual representation is manageable with respect to the polytopes.

Thus, the optimization problem (35) takes the form:

$$
\begin{array}{rl}
\min _{\Delta_{j}, c_{j}} & \mathcal{C}\left(\Delta_{j}, c_{j}\right) \\
\text { s.t. } & P_{j} \subseteq \mathcal{Z}\left(G \Delta_{j}, c_{j}\right) \\
& \mathcal{Z}\left(G_{j} \Delta_{j}, c_{j}\right) \subset \mathcal{R}_{i j}^{ \pm}, \forall i .
\end{array}
$$

As was stated above, the choice between $\mathcal{R}_{i j}^{-}$and $\mathcal{R}_{i j}^{+}$ (42b) depends on position of the center. The constraint (42b) is formulated recalling the inclusion condition of a parametrized zonotope into a polytope (34).

Theorem 2: Corridor constraints are convex with respect to the zonotopic over-approximations. 
Proof: Taking into account that the separation constraints (42b) are linear, the convexity of the feasible space regarding zonotopic over-approximation is straightforward

Remark 8: Certainly, (42b) enforces the corridors but it represents an additional structural constraint and impacts the feasiblity of the zonotopic over-approximations. For example, let us consider the case depicted in the Fig. 4, where we take a triangle in $\mathbb{R}^{2}$ and proceed to over-approximate with a parametrized zonotope with $G$ given by the Euclidean unit vectors. The problem (42) is infeasible.

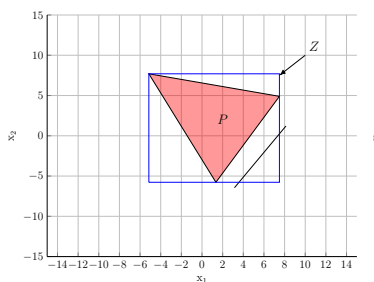

(a) infeasible for $G=G_{2}$

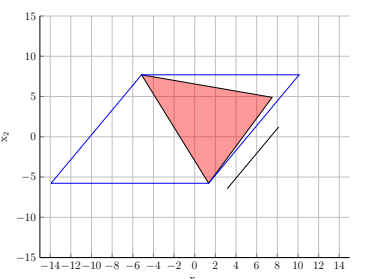

(b) feasible for $\left[\begin{array}{ll}G_{2} & Q_{1}\end{array}\right]$
Fig. 4: The feasibility of (42).

Despite the limitation highlighted in Remark 8, there exists a strong constructive result for the existence of a zonotopic over-approximation which satisfies the corridor constraints.

Proposition 3: Any polyhedral set (1) can be approximated by a zonotopic set (23) with corridor type of constraints (41) by an adequate choice of generators $G$.

Proof: The idea is to generate a face of the zonotopic overapproximation aligned to the separating hyperplane. This is done by spanning the separation hyperplane with a subset of the generators. More precisely, we should obtain any basis for the subspace described by the separating hyperplane. This basis represents a valid subset of generators for the zonotopic set. The result is constructive if we consider a full rank orthogonal matrix $Q$ with $Q=\left[\begin{array}{ll}h_{\text {sep }}^{\top} & Q_{1}\end{array}\right]$. If $G$ includes a basis of the space perpendicular on separating hyperplane, i.e. $Q_{1}$ then the problem (42) is feasible (as in Fig. 4b).

A key issue with this approach is that we cannot guarantee the monotonicity of the over-approximations with respect to the number of the generators. By adding a generator as in the proof of Corollary 3, we solve an obstructed corridor and, at the same time, we may obstruct others which were initially unobstructed. An important theoretical result which guarantees the separation in a $d$-dimensional space for a number of " $d+1$ " over-approximations is presented next.

Theorem 3: In $\mathbb{R}^{d}$, the maximum number of joint constraints for corridors with feasibility guarantees is $d+1$. Proof: Let $P_{1}, \ldots, P_{d+1}$ be the obstacles and $P_{i}$ a generic element of this collection. If $P_{i} \cap P_{j}=\emptyset, \forall i \neq j$, then there exist $d$ separation hyperplanes $h_{i j}$ and a zonotope satisfying $w_{i j} \leq\left\|h_{i j}^{\top} x\right\| \leq \overline{w_{i j}}, \forall x \in P_{i}$. Obviously, $w_{i j}$ and $\overline{w_{i j}}$ can be chosen in order to avoid the intersection with one side of the separation hyperplane.

Corollary 2: For any " $d+1$ " obstacles, there exists a "box" approximation which guarantees the separation.
Proof: Firstly, we construct the matrix $G$ such that the resulting over-approximation to have the facets parallel with the " $d+1$ " separating hyperplanes (for each separating hyperplane we choose the vectors which span the space perpendicular on that hyperplane). There exists a zonotopic over-approximation which can be adjusted either through a proper scaling factor or through a displacement of the center.

\section{Illustrative example}

We depicted in Fig. 5 the results of a procedure which combines the advantages given by Theorem 2 and Corollary 3. This procedure performs well in simulations, but it cannot provide theoretical guarantees on the separation among the zonotopic over-approximations within a generic multi-obstacle environment.

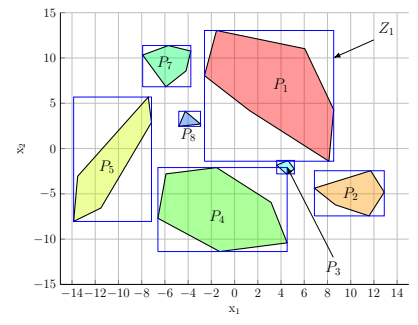

(a) initial

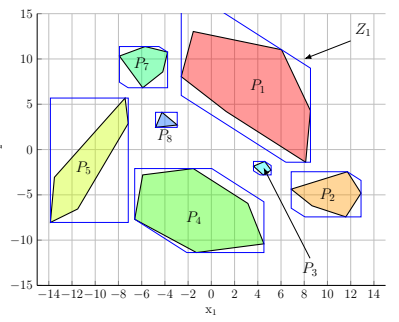

(b) after
Fig. 5: Maintaining corridors procedure over example in Fig. 3

As was stated above, the strong result from Theorem 3 is restricted to $d+1$ obstacles. In order to cope with a generic obstacles distribution (in terms of their number and positions) we use a strategy able to select these $d+1$ obstacles for each given state based on the results from Section III and on the prototype MPC strategy from Section IV-A.

\section{Generic collision avoidance strategy and examples}

The proposed motion planning strategy through a multiobstacle environment can be summarized by the following steps:

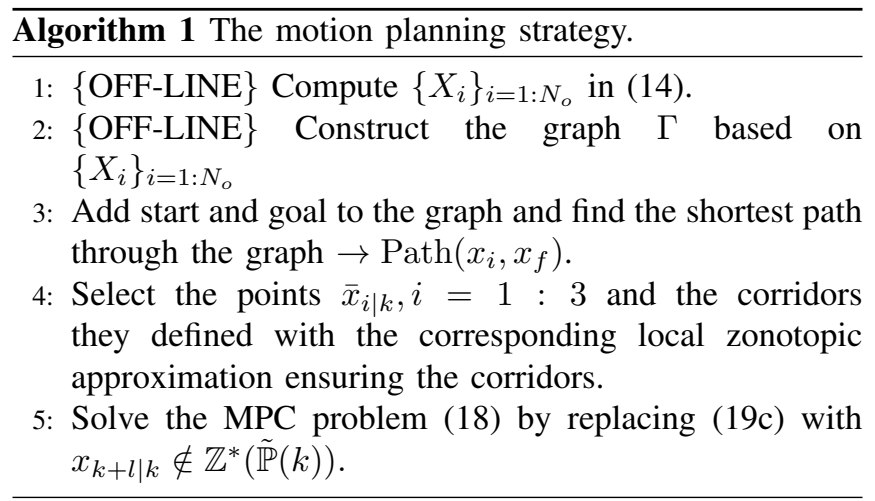

For illustration, let us consider an agent described by the dynamics (5a) in $\mathbb{R}^{d}$ :

$$
A=\left[\begin{array}{cc}
O_{d} & I_{d} \\
O_{d} & -\frac{\mu}{M} I_{d}
\end{array}\right], \quad B=\left[\begin{array}{c}
O_{d} \\
M I_{d}
\end{array}\right],
$$


where $\mu=3$ and $M=60$. The agent's state and input are constrained: $\mathcal{X}=\left\{x:-15 \leq x_{i} \leq 15, \forall i=1 \ldots 2 d\right\}$ and $\mathcal{U}=\left\{u:-1 \leq u_{i} \leq 1, \forall i=1 \ldots 2 d\right\}$.

In order to obtain a collision free trajectory for the agent through the complex ${ }^{4}$ multi-obstacle environment depicted in Fig. 2 we firstly employed the strategy (4) with enhancement (10), considering the entire set of obstacles and imposing a large prediction horizon $\left(N_{p}=40\right)$. The resulting trajectory (the red one from Fig. 6a) did not converge to the final position, the agent remaining on the boundary of an obstacle. However, applying the Algorithm 1 we obtain a trajectory, which attains $x_{f}$. Moreover, Fig. $6 \mathrm{~b}$ depicts the local zonotopic approximation corresponding to the first sequence of $\bar{x}_{i \mid k}, i=1: 3$, and the highlighted obstacles represent the union of all sets $\tilde{\mathbb{P}}(k)$ whose approximations were used in the control problem at a certain moment.

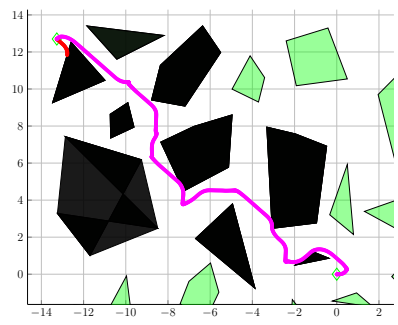

(a) Trajectories

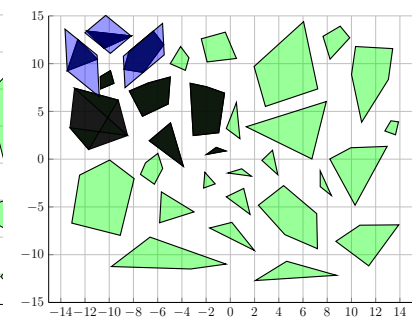

(b) $\mathbb{Z}^{*}(\tilde{\mathbb{P}}(k))$
Fig. 6: Simulation results for the environment Fig. 2

\section{CONCLUSIONS}

The obstacle avoidance problem was treated aiming to ensure the convergence and feasibility and, concurrently, to reduce the complexity of the original problem. Based on convex lifting and graph theory we have generated feasible reference trajectories which were furthered employed in the collision avoidance strategy. Additionally, zonotopic overapproximations were used to cover efficiently the non-convex region of interest and a series of theoretical results were proposed to address the issues related to their impact on the control problem.

\section{APPENDIX}

Volume of the parametrized zonotopes

The zonotopes volume has an explicit formulation [24]:

$$
\operatorname{Vol}(\mathcal{Z}(G, c))=\sum_{1 \leq j_{1}<j_{2} \ldots j_{d} \leq m}\left|\operatorname{det}\left(G^{j_{1} \ldots j_{d}}\right)\right|
$$

where $G^{j_{1} \ldots j_{d}}$ denotes the matrix composed from columns of indices $j_{1} \ldots j_{d}$ from $G$. Replacing $g_{k}$ with $g_{k} \cdot \delta_{j_{k}}$ in (43) leads to

$$
\operatorname{Vol}\left(\mathcal{Z}\left(G \Delta_{j}, c_{j}\right)\right)=\sum_{1 \leq k_{1}<\cdots<k_{d} \leq m}\left|\operatorname{det}\left(G^{k_{1} \ldots k_{d}} \cdot \Delta_{j}^{k_{1} \ldots k_{d}}\right)\right| .
$$

Noting that $\operatorname{det}(M \cdot N)=\operatorname{det}(M) \cdot \operatorname{det}(N)$ and that $\Delta_{j}$ is a diagonal matrix with positive elements gives (36).

\footnotetext{
${ }^{4}$ in the sense of the number of the obstacles, $N_{o}=33$
}

\section{REFERENCES}

[1] F. Janecek, M. Klauco, M. Kaluz, and M. Kvasnica, "OPTIPLAN: A Matlab Toolbox for Model Predictive Control with Obstacle Avoidance," IFAC-PapersOnLine, vol. 50, no. 1, pp. 531-536, Jul. 2017.

[2] S. Manyam, D. Casbeer, and K. Sundar, "Path planning for cooperative routing of air-ground vehicles," arXiv preprint:1605.09739, 2016.

[3] A. Richards and J. P. How, "Aircraft trajectory planning with collision avoidance using mixed integer linear programming," in American Control Conference, 2002. Proceedings of the 2002, vol. 3. IEEE, 2002, pp. 1936-1941.

[4] I. Prodan, F. Stoican, S. Olaru, and S.-I. Niculescu, "Mixed-Integer Representations," in Mixed-Integer Representations in Control Design, ser. SpringerBriefs,Engineering. Springer, Cham, 2016.

[5] Y.-b. Chen, G.-c. Luo, Y.-s. Mei, J.-q. Yu, and X.-1. Su, "UAV path planning using artificial potential field method updated by optimal control theory," International Journal of Systems Science, vol. 47, no. 6, pp. 1407-1420, Apr. 2016.

[6] R. Olfati-Saber and R. M. Murray, "Distributed cooperative control of multiple vehicle formations using structural potential functions," in IFAC world congress, vol. 15, no. 1, 2002, pp. 242-248.

[7] J.-C. Latombe, Robot motion planning. Springer Science \& Business Media, 2012, vol. 124.

[8] S. Karaman and E. Frazzoli, "Sampling-based algorithms for optimal motion planning," The International Journal of Robotics Research, vol. 30, no. 7, pp. 846-894, Jun. 2011.

[9] S. M. LaValle, "Rapidly-exploring random trees: A new tool for path planning," Tech. Rep., 1998.

[10] M. P. Vitus, S. L. Waslander, and C. J. Tomlin, "Locally optimal decomposition for autonomous obstacle avoidance with the tunnelmilp algorithm," in Decision and Control, 2008. CDC 2008. 47th IEEE Conference on. IEEE, 2008, pp. 540-545.

[11] I. Prodan, F. Stoican, S. Olaru, and S.-I. Niculescu, "Enhancements on the hyperplanes arrangements in mixed-integer programming techniques," Journal of Optimization Theory and Applications, vol. 154, no. 2, pp. 549-572, 2012.

[12] J. P. Vielma and G. L. Nemhauser, "Modeling disjunctive constraints with a logarithmic number of binary variables and constraints," Mathematical Programming, vol. 128, no. 1-2, pp. 49-72, 2011.

[13] F. Stoican, I. Prodan, and S. Olaru, "Hyperplane arrangements in mixed-integer programming techniques. Collision avoidance application with zonotopic sets," in 2013 European Control Conference (ECC), Jul. 2013, pp. 3155-3160.

[14] L. J. Guibas, A. Nguyen, and L. Zhang, "Zonotopes As Bounding Volumes," in Proceedings of the Fourteenth Annual ACM-SIAM Symposium on Discrete Algorithms, ser. SODA '03. Philadelphia, PA, USA: SIAM, 2003, pp. 803-812.

[15] M. Althoff, O. Stursberg, and M. Buss, "Computing reachable sets of hybrid systems using a combination of zonotopes and polytopes," Nonlinear analysis: hybrid systems, vol. 4, no. 2, pp. 233-249, 2010.

[16] V. Puig, "Fault diagnosis and fault tolerant control using setmembership approaches: Application to real case studies," International Journal of Applied Mathematics and Computer Science, vol. 20, no. 4, pp. 619-635, 2010.

[17] T. Alamo, J. M. Bravo, and E. F. Camacho, "Guaranteed state estimation by zonotopes," Automatica, vol. 41, no. 6, pp. 1035-1043.

[18] M. Althoff, "Cora 2015 manual," TU Munich, vol. 85748, 2015.

[19] G. M. Ziegler, Lectures on polytopes. Springer Science \& Business Media, 2012, vol. 152.

[20] N. A. Nguyen, M. Gulan, S. Olaru, and P. Rodriguez-Ayerbe, "Convex lifting: Theory and control applications," IEEE Transactions on Automatic Control, vol. 63, no. 5, pp. 1243-1258, 2018.

[21] W. Kuhn, "Rigorously computed orbits of dynamical systems without the wrapping effect," Computing, vol. 61, no. 1, pp. 47-67, Mar. 1998.

[22] K. Fukuda, "From the zonotope construction to the Minkowski addition of convex polytopes," Journal of Symbolic Computation, vol. 38, pp. 1261-1272, Oct. 2004.

[23] I. Kolmanovsky and E. G. Gilbert, "Theory and computation of disturbance invariant sets for discrete-time linear systems," Mathematical problems in engineering, vol. 4, no. 4, pp. 317-367, 1998.

[24] E. Gover and N. Krikorian, "Determinants and the volumes of parallelotopes and zonotopes," Linear Algebra and its Applications, vol. 433, no. 1, pp. 28-40, Jul. 2010. 